\title{
BMJ Open Economic costs of infertility care for patients in low-income and middle- income countries: a systematic review protocol
}

\author{
Purity Njagi (D) , ${ }^{1}$ Wim Groot, ${ }^{1,2}$ Jelena Arsenijevic, ${ }^{3}$ Silke Dyer, ${ }^{4}$ Gitau Mburu, ${ }^{5}$ \\ James Kiarie ${ }^{5}$
}

To cite: Njagi P, Groot W, Arsenijevic J, et al. Economic costs of infertility care for patients in low-income and middle-income countries: a systematic review protocol. BMJ Open 2020;10:e042951. doi:10.1136/ bmjopen-2020-042951

- Prepublication history for this paper is available online. To view these files, please visit the journal online (http://dx.doi. org/10.1136/bmjopen-2020042951).

Received 20 July 2020 Revised 11 0ctober 2020 Accepted 28 October 2020

A) Check for updates

(c) Author(s) (or their employer(s)) 2020. Re-use permitted under CC BY-NC. No commercial re-use. See rights and permissions. Published by BMJ.

For numbered affiliations see end of article.

Correspondence to

Purity Njagi;

p.njagi@student.

maastrichtuniversity.nl

\section{ABSTRACT}

Introduction Infertility, a condition of the reproductive system, affects millions of individuals and couples worldwide. Despite infertility treatment's existence, it is largely unavailable and inaccessible in low/middle-income countries (LMICs) due to the prohibitive costs compounded by an absence of financing. Previous systematic reviews have shown that there is scanty information in LMICs on out-of-pocket (0OP) payments for infertility treatment. This protocol outlines the methodological approach and analytical process to appraise the extent of economic burden due to payments for infertility care services in LMICs.

Method and analysis Using the Preferred Reporting Items for Systematic Reviews and Meta-Analyses approach, we will primarily search for articles indexed in PubMed, Web of Science, Cumulative Index of Nursing and Allied Health Literature, EconLit and PsycINFO databases. Grey literature from relevant organisations' virtual libraries shall also be searched. Backward and forward searches on the articles selected will also be done. Quantitative studies on infertility treatment costs from LMICs across the world regions within the last 20 years will be considered. The primary outcome of interest shall include 00P payments, catastrophic health expenditure and direct costs for infertility services. Conversely, informal payments and indirect costs related to infertility treatments shall be considered as secondary outcomes. Integrated quality Criteria for Review Of Multiple Study designs will be used to assess the quality of the studies included in the review. Meta-analysis shall be considered if sufficient studies identified are homogenous in characteristics. Also, the review shall analyse the average cost of infertility treatment against the respective countries' economic indicators like gross domestic product per capita if data permit.

Ethics and dissemination Research and ethics approval will not be required given this will be a review of published articles on the subject. The findings shall be disseminated through publication in a peer-reviewed journal and presentation to the $\mathrm{WHO}$ and its partners. PROSPERO registration number CRD42020199312.

\section{BACKGROUND}

Infertility is a condition of the reproductive system defined as the failure to achieve a
Strengths and limitations of this study

This review will synthesise information on the economic costs of infertility care for patients or consumers in low/middle-income countries in accordance with the Preferred Reporting Items for Systematic Reviews and Meta-Analyses guidelines.

- The review will search journals from multidisciplinary fields and grey literature and will include non-English studies to optimise on the breadth of literature across all world regions.

- The review will show any potential correlation between the infertility costs and the context of the healthcare system and/or living standards in the respective countries.

- The results from this review will contribute to enhanced visibility and advocacy about the economic costs faced by individuals and couples with infertility.

- Heterogeneity of the studies and outcomes could limit the interpretation of the results reported.

clinical pregnancy after 12 months or more of regular unprotected sexual intercourse. ${ }^{1}$ It is attributed to anomalies in either male or female reproductive systems, and in some cases, the cause may be unexplained. ${ }^{23}$ Infertility is either primary or secondary, whereby primary infertility refers to situations where an individual has never achieved a clinical pregnancy while secondary infertility refers to situations where a clinical pregnancy has been achieved at least once before. ${ }^{1}$

Globally, infertility affects millions of individuals and couples. However, the estimates vary widely with some studies reporting as high as 120 million women, ${ }^{4}$ while others report lower estimates of about 48.5 million couples. ${ }^{5}$ The prevalence of infertility ranges between $8 \%$ and $12 \%$ worldwide, with relatively higher prevalence rates in lessdeveloped countries/regions such as subSaharan Africa, South Asia, North Africa/ Middle East, and Central/Eastern Europe 
and Central Asia. ${ }^{6}$ The geographical differences are influenced by environmental, cultural and societal factors. ${ }^{7}$

Infertility can have significant public health, psychological and social consequences. It is associated with depression, anxiety, isolation, loss of control, ${ }^{8}$ violence, marital breakdown and divorce. ${ }^{9}$ In many pro-natalist societies, the infertility burden falls disproportionately on women, who are often marginalised, socially excluded and stigmatised. ${ }^{10}$

Treatment for infertility provides an opportunity for women and men to become parents. The evolution of assisted reproductive technology (ART) for the treatment of infertile couples is considered an extraordinary restorative accomplishment throughout the world. ${ }^{11}$ Many women and couples have been able to conceive due to the advancements in reproductive technology. ${ }^{12}$ Access to diagnosis and treatment of infertility reduces social inequities and emotional difficulties. ${ }^{13}$ However, infertility treatment can be costly, time-consuming, strenuous and frustrating - or outright inaccessible in the first instance. $^{1415}$

In addition, there are marked disparities in availability, quality and delivery of infertility care services between developed and less-developed countries. ${ }^{16}$ Despite the existence of ART for nearly three decades, it is either unavailable or inaccessible to most people in resource-poor countries. ${ }^{17-19}$ There is a growing need for infertility treatment such as in vitro fertilisation (IVF) in low/middle-income countries (LMICs), but the demand outstrips availability, and the costs are prohibitive. ${ }^{1720}$ Moreover, infertility in many resource-limited settings such as sub-Sahara Africa is often overlooked due to many competing health needs, as well as the relatively high fertility rates and large family sizes which may mask infertility in populations. ${ }^{21}$ As a result, in many LMICs, publicly funded infertility treatments are either limited or non-existent and are excluded from health insurance packages. ${ }^{22}$ The limited financial insurance for ART treatments, although the associated high costs, implies that infertility is viewed as a condition underserving of financial assistance in LMICs. ${ }^{23}$

Consequently, the high cost of care borne by individuals is reported among the key barriers to accessing assisted reproduction services. ${ }^{24}$ In LMICs infertility services are generally accessed by the well-off, at mainly private health facilities. ${ }^{25}$ Accessibility of treatments such as IVF is limited to only those who can afford to pay out of pocket (OOP) ${ }^{26}$ Even among those who have the ability to pay, their willingness and financial ability to undergo multiple cycles of ART often depends on OOP payments incurred. ${ }^{27}$ However, due to the desire for a child, couples are often ready to make significant financial sacrifices often beyond their means. ${ }^{28}$ Couples are frequently willing to suffer catastrophic financial hardship instead of forgoing infertility care, resulting in negative economic consequences. ${ }^{29}$ Evidence suggests that in the absence of mechanisms for risk protection, OOP payments can push households into poverty. ${ }^{30}$
Despite evidence that infertility treatment can cause or exacerbate economic inequalities, public financing of fertility services is often neglected in LMICs. ${ }^{7}$ The lack of appropriate financial protection systems in LMICs could be linked to the fact that the majority of the economic studies of infertility have been conducted in high-income countries. ${ }^{31}$ This is perhaps because infertility treatment is more established, and there exist some mechanisms for financing. ${ }^{32}$ On the contrary, decision making on infertility in LMICs is constrained by the limited availability of reliable evidence regarding current needs. ${ }^{33}$ Although specific costs may vary across countries based on laws, regulations and insurance coverage for assisted reproduction, ${ }^{34}$ a better understanding of the economic consequences of infertility is needed to inform financial protection policies in LMICs.

In 2012, Dyer and Patel ${ }^{35}$ conducted a systematic review of the economic impact of infertility in developing countries that found a significant risk of catastrophic payments due to infertility treatment costs borne by households. However, the review found that there was scanty information on OOP and other economic data about infertility care in developing countries. Another gap is related to the need to contextualise OOP and other costs with economic parameters such as gross domestic product (GDP) of the countries where such costs were incurred, which was not addressed in the above review. This is particularly relevant given the argument that the establishment of infertility services in developing countries depends on the healthcare system and stage of development of the country. ${ }^{36}$ Therefore, a pertinent need to both appraise the current status of the financial burden due to payment for infertility services, and to also relate the financial burden to the varying healthcare and economic contexts within which infertility services are offered in LMICs.

\section{Aim}

This systematic literature review aims to provide evidence on the extent of the financial burden imposed by costs incurred in seeking infertility care services and establish any potential correlation with the economic indicators in LMICs.

\section{Objectives}

This review is intended to:

1. Assess the extent of the financial burden imposed on individuals or couples or households who seek infertility treatment services in LMICs.

2. Examine the association of the financial burden due to infertility treatment against the respective countries' healthcare context or macroeconomic indicators (eg, GDP/income per capita).

\section{METHODS AND ANALYSIS}

To enhance transparency, this systematic review protocol has been developed according to the Preferred Reporting Items for Systematic Review and Meta-Analysis Protocols (PRISMA-P). ${ }^{37}$ 


\section{Design}

The systematic review will be conducted and reported according to the Preferred Reporting Items for Systematic Reviews and Meta-Analyses: the PRISMA statement. ${ }^{38}$

\section{Search strategy}

The review will search articles indexed in the following databases: PubMed, Cumulative Index of Nursing and Allied Health Literature, Web of Science, EconLit and PsycINFO. Reference lists from identified articles and reviews will further be searched to locate additional studies.

The search for peer-reviewed manuscripts will be complemented with a grey literature search which shall include a systematic search on Google Scholar, and a search on virtual libraries of relevant organisations like WHO and other institutions such as the International Federation of Gynaecology and Obstetrics, International Federation of Fertility Societies, International Committee for Monitoring Assisted Reproductive Technologies and the Demographic and Health Surveys Program. Proceedings and abstracts from the following conferences will be searched: European Society of Human Reproduction and Embryology, the American Society for Reproductive Medicine, Latin American Network of Assisted Reproduction and Asia Pacific Initiative on Reproduction. In addition, a forward and backward reference search of authors mentioned in selected articles, and a purposive search of authors who have published prominently in this area, will be conducted.

\section{Search string of keywords}

The search terms shall include:

1. 'Infertility' OR 'subfertility' OR 'Sterility' OR 'Fecundity' OR 'Subfecundity' OR 'Childlessness' OR 'Assisted reproduction technology (ART)' OR 'Intra vitro fertilization (IVF)', 'Intrauterine insemination (IUI)', 'intra-cytoplasmic sperm-insemination (ICSI) AND;

2. 'Costs' OR 'Out-of-pocket' OR 'Payments' OR 'Feefor-service' OR 'OR 'Expenditure' OR 'Financial burden' OR 'Economic' AND;

3. 'Low-income countries' OR 'Middle-income countries' OR 'Developing countries' OR 'Less-developed countries' OR 'Least developed countries' OR 'Africa' OR 'Asia' OR 'Latin America' OR 'Caribbean' OR 'Middle East' OR 'Eastern Europe'.

LMIC filter (https://epoc.cochrane.org/lmic-filters) shall be used for PubMed.

\section{Inclusion/exclusion criteria \\ Study design}

Articles will be included regardless of their study design which may consist of cross-sectional studies, case-control, comparative or longitudinal/cohort studies. Studies will not need to have a comparative arm to be included. Qualitative studies will be considered if they include summaries of healthcare costs associated with infertility care. Discussion papers, policy briefs, guidelines and programme reports will be excluded from this review.

\section{Population}

The review shall include studies that focus on all population groups provided they are at risk of or have experienced infertility.

\section{Study setting}

The geographical focus of the review will be restricted to studies undertaken in LMICs as defined by the World Bank (http://data.worldbank.org/country). The World Bank classifies countries according to their gross national income per capita into low-income, lower/middleincome, upper/middle-income and high-income countries. LMICs are sometimes referred to as developing countries.

\section{Outcome of interest}

Studies will be included if they assessed the direct costs (eg, consultation, medication) and indirect costs (eg, transport, post-treatment care) associated with diagnosis, treatment, monitoring and post-treatment of infertility. This will be categorised into both primary and secondary outcomes of interest.

\section{Primary outcomes}

The primary outcomes of this review shall include estimates of costs associated with infertility treatment presented as either:

1. Catastrophic Health Expenditure (CHE), defined as any expenditure for health treatment that threatens a household's financial ability to maintain its subsistence needs. CHE does not necessarily refer to high costs. The review shall extract and report on the respective thresholds as applied in the respective articles assessed.

2. OOP payments shall refer to direct payments made by individuals to healthcare providers to seek treatment for infertility services or assisted reproduction. The review shall assess not only the average costs for the entire treatment but also the cost per cycle if those data are available or provided.

3. Direct costs for infertility services as classified in primary papers, and this is expected to include expenses such as consultations, examinations, treatments, medicines and hospitalisation.

\section{Secondary outcomes}

The secondary outcome(s) shall include:

1. Informal payments, which shall refer to payments to individual and institutional providers, in kind or in cash, that are made outside official payment channels or are purchases meant to be covered by the healthcare system. $^{39}$

2. Other indirect costs associated with assisted reproduction or infertility treatment, including expenses such as accommodation before and after treatment, travel for treatment and cost of follow-up care after the initial consultation. 
The review will seek to establish the costs by type of treatment options if the information is available. In addition, the total average cost for the infertility services shall be mapped against study country context of the healthcare system (for instance presence or absence of financing for infertility services) or the GDP per capita of the respective country.

\section{Years of publication}

The review shall consider studies published within the last 20 years (2000-2020). Older studies will be excluded given that a previous systematic review ${ }^{35}$ revealed that studies conducted in the 1990s were generally qualitative and may be less informative for the current situation. Hence, this review will focus on the most recent publications from the last two decades.

\section{Language of publication}

The initial search of this review will include all studies irrespective of language. The title, and if required the abstract, of non-English publications, will be translated using Google Translate for the first screening phase. Consequently, non-English papers within the scope of this review will be translated in full using Google Translate. If necessary and possible, further means of the translation will be sought. The main author of non-English publications will be contacted by email to verify and corroborate the English translation of their work and the main findings. Reminders will be sent to the main author and if necessary, to coauthors.

\section{Screening and selection of studies}

Two researchers will independently review the titles and abstracts of all identified studies. Studies will be included based on the above criteria. Any potential conflicts that arise on whether to include or exclude a study will be resolved through consensus and the reasons for exclusion documented. A flow diagram illustrating the literature search, article selection and final included studies will be generated per the PRISMA guidelines for systematic reviews. Definitive studies for inclusion in the final evaluation shall be identified and agreed on by all the reviewers.

\section{Data extraction}

Data will be extracted by two main reviewers and crosschecked for accuracy and consistency by a third reviewer independently. Any discrepancies will be resolved and agreed through a discussion with a third reviewer. Should there be a need for clarification on published papers, authors of original papers shall be contacted to provide additional information for any missing or unclear data. If such an author does not provide the requested clarification within 4 weeks, the review team will determine whether to include the study in the review based on the impact of the missing information on the team's ability to draw necessary conclusions.

In general, the review will extract the following information from each study: author, year of publication, study title, study objectives, study design, country/ region, subnational (an area, region, state or province in a country), or national level, population type, sample sizes, the primary outcome of interest and the secondary outcomes. A matrix table will be used to summarise the characteristics of the included studies.

\section{Quality assessment of included studies}

We recognise that the quality of the systematic review is dependent on the quality appraisal of the information captured from the respective studies reviewed. ${ }^{40}$ In light of this, the methodological quality (or risk of bias) of the individual studies eligible for a full assessment will be appraised using the Integrated quality Criteria for Review Of Multiple Study designs (ICROMS). ${ }^{41}$ ICROMS provides for quality appraisal of a broad range of study designs as anticipated in this review. The tool has been applied to assess validity in other systematic reviews. ${ }^{42}$ Two reviewers will independently complete the quality assessment, and any discrepancies will be discussed and resolved by consultation with a third reviewer.

\section{Analysis and reporting}

An overview of the selection process will be presented in accordance with the PRISMA flow chart summarising the identification, screening and eligibility process. A narrative synthesis will summarise and explain the characteristics and findings of the studies. Tables will be used to provide a summary of the data extracted from the individual studies assessed. Findings of the review will be appraised, synthesised and submitted for publication in a peer-reviewed journal.

Given the expected heterogeneous nature of the outcomes of interest and potential quality of the outcome variables, this review shall primarily focus on the descriptive analysis. However, meta-analysis will be considered if several quantitative studies identified are homogenous in characteristics including study design and outcome of interest, and if there are sufficient outcome estimates to perform statistical analysis.

If the review produces a sufficient number of studies for meta-analysis, then sensitivity analysis will be conducted to assess the impact of the quality of the article, bias and sample size.

\section{Patient and public involvement}

Although the beneficiary is important in research, patients were not involved in the development of this systematic review protocol.

\section{ETHICS AND DISSEMINATION}

The results of the review are expected to inform advocacy for infertility treatment in various resource-limited settings. The findings shall, therefore, be published in a relevant journal. In addition, the results will be disseminated through presentations forum at relevant scientific meetings, mass emails, social media, and the website of the WHO Sexual and Reproductive Health and Research 
department. Other potential forums where these results can be presented to relevant stakeholders shall be identified.

\section{DISCUSSION AND EXPECTED IMPACT}

The findings from this review are expected to highlight the magnitude of the financial burden related to infertility care across different geographical regions. This review is also expected to show any possible correlation between the infertility costs and the context of the country's healthcare system, for instance, the presence of financing for infertility services or economic markers like GDP or income per capita. Understanding the economic costs of infertility care will contribute to formulating of appropriate policies that enable individuals having infertility to access care or treatment in LMICs.

It is possible that multiple pregnancies, which tend to be costly to health systems and households, may result from ART in contexts where single embryo transfer may not always be the norm. To the extent possible, data will be explored to identify if costs reported were reported in the context of multiple pregnancies. However, a comprehensive assessment of downstream cost of pregnancy and following delivery, be it singleton or multiples, is outside the scope of this review, and would be best addressed in a separate review.

Results of this review will also contribute to enhanced visibility and advocacy to eliminate high economic costs that face individuals and couples with infertility and subfertility. This is particularly relevant given that provision of fertility services is an integral component of comprehensive sexual and reproductive health services that are needed to attain the Sustainable Development Goal no. 3 (Ensure healthy lives and promote well-being for all at all ages).

\section{LIMITATIONS}

We envision a few limitations in undertaking this review, including non-standard measures of the primary outcome of interests (financial burden) across the studies. Heterogeneity of the studies and computation of the outcome(s) could limit the interpretation of the results reported. Nevertheless, this review shall capture and analyse the financial burden as reported in the studies as this may provide nuances on how this is measured and interpreted across countries.

\section{Author affiliations}

${ }^{1}$ Maastricht Graduate School of Governance, United Nations University-MERIT/ Maastricht University, Maastricht, Netherlands

${ }^{2}$ Department of Health Services Research, Faculty of Health, Medicine and Life Sciences, Maastricht University, Maastricht, Netherlands

${ }^{3}$ School of Governance, Faculty of Law, Economics and Governance, Utrecht University, Utrecht, Netherlands

${ }^{4}$ Department of Obstetrics and Gynaecology, University of Cape Town, Cape Town, South Africa

${ }^{5}$ Department of Sexual and Reproductive Health and Research (SHR), World Health Organization, Geneve, Switzerland
Contributors PN and GM conceptualised the review idea and drafted the protocol. WG and JA reviewed the protocol and provided advice on the methodological approach and the analytical process. SD, GM and JK provided advice on conceptualisation of the review and content oversight. All authors have approved the publication of the protocol.

Funding This work was supported by the WHO, Department of Sexual and Reproductive Health and Research (SHR).

Competing interests None declared.

Patient and public involvement Patients and/or the public were not involved in the design, or conduct, or reporting, or dissemination plans of this research.

Patient consent for publication Not required.

Provenance and peer review Not commissioned; externally peer reviewed.

Open access This is an open access article distributed in accordance with the Creative Commons Attribution Non Commercial (CC BY-NC 4.0) license, which permits others to distribute, remix, adapt, build upon this work non-commercially, and license their derivative works on different terms, provided the original work is properly cited, appropriate credit is given, any changes made indicated, and the use is non-commercial. See: http://creativecommons.org/licenses/by-nc/4.0/.

ORCID iD

Purity Njagi http://orcid.org/0000-0003-3157-4413

\section{REFERENCES}

1 Zegers-Hochschild F, Adamson GD, Dyer S, et al. The International glossary on infertility and fertility care, 2017. Fertil Steril 2017;108:393-406.

2 Benksim A, Elkhoudri N, Addi RA, et al. Difference between primary and secondary infertility in Morocco: frequencies and associated factors. Int J Fertil Steril 2018;12:142-6.

3 Podolska MZ, Bidzan M. Infertility as a psychological problem. Ginekol Pol 2011;82:44-9.

4 Boivin J, Bunting L, Collins JA, et al. International estimates of infertility prevalence and treatment-seeking: potential need and demand for infertility medical care. Hum Reprod 2007;22:1506-12.

5 Mascarenhas MN, Flaxman SR, Boerma T, et al. National, regional, and global trends in infertility prevalence since 1990: a systematic analysis of 277 health surveys. PLoS Med 2012;9:e1001356.

6 Inhorn MC, Patrizio P. Infertility around the globe: new thinking on gender, reproductive technologies and global movements in the 21st century. Hum Reprod Update 2015;21:411-26.

7 Ombelet W. Global access to infertility care in developing countries: a case of human rights, equity and social justice. Facts Views Vis Obgyn 2011;3:257-66.

8 Rooney KL, Domar AD. The relationship between stress and infertility. Dialogues Clin Neurosci 2018;20:41-7.

9 Agarwal N, Marks M. For better or worse? Fertility challenges and marital dissolution 2019.

10 Tiu MM, Hong JY, Cheng VS, et al. Lived experience of infertility among Hong Kong Chinese women. Int J Qual Stud Health Wellbeing 2018;13:1554023.

11 Sharma RS, Saxena R, Singh R. Infertility \& assisted reproduction: A historical \& modern scientific perspective. Indian J Med Res 2018;148:S10-14.

12 Bitler MP, Schmidt L. Utilization of infertility treatments: the effects of insurance mandates. Demography 2012;49:125-49.

13 Makuch MY, Simônia de Padua K, Petta CA, et al. Inequitable access to assisted reproductive technology for the low-income Brazilian population: a qualitative study. Hum Reprod 2011;26:2054-60.

14 Domar A, Gordon K, Garcia-Velasco J, et al. Understanding the perceptions of and emotional barriers to infertility treatment: a survey in four European countries. Hum Reprod 2012;27:1073-9.

15 Rouchou B. Consequences of infertility in developing countries. Perspect Public Health 2013;133:174-9.

16 Nachtigall RD. International disparities in access to infertility services. Fertil Steril 2006;85:871-5.

17 Allahbadia GN. Ivf in developing economies and low resource countries: an overview. J Obstet Gynaecol India 2013;63:291-4.

18 Sharma S, Mittal S, Aggarwal P. Management of infertility in low resource countries. BJOG 2009;116 Suppl 1:77-83.

19 Botha B, Shamley D, Dyer S. Availability, effectiveness and safety of art in sub-Saharan Africa: a systematic review. Hum Reprod Open 2018;2018:hoy003. 
20 Ombelet W, Onofre J. IVF in Africa: what is it all about? Facts Views Vis Obgyn 2019;11:65-76.

21 Asemota O, Klatsky P. Access to infertility care in the developing world: the family promotion gap. Semin Reprod Med 2015;33:017-22

22 Adewumi EA. Infertility treatment financing in Nigeria. Nigerian Journal of Health Sciences 2017;17:38.

23 Insogna IG, Ginsburg ES, Infertility GES. Infertility, inequality, and how lack of insurance coverage compromises reproductive autonomy. AMA J Ethics 2018;20:E1152-9.

24 Casebolt MT. Barriers to reproductive health services for women with disability in low- and middle-income countries: a review of the literature. Sex Reprod Healthc 2020;24:100485.

25 Hall DR, Hanekom G. Assisted reproduction and justice: threats to a new model in a low- and middle-income country. Dev World Bioeth 2020;20:167-71.

26 Teoh PJ, Maheshwari A. Low-cost in vitro fertilization: current insights. Int J Womens Health 2014;6:817-27.

27 Wu AK, Odisho AY, Washington SL, et al. Out-Of-Pocket fertility patient expense: data from a multicenter prospective infertility cohort. J Urol 2014;191:427-32.

28 Dyer SJ, Vinoos L, Ataguba JE. Poor recovery of households from out-of-pocket payment for assisted reproductive technology. Hum Reprod 2017;32:2431-6.

29 Dyer SJ, Sherwood K, Mclntyre D, et al. Catastrophic payment for assisted reproduction techniques with conventional ovarian stimulation in the public health sector of South Africa: frequency and coping strategies. Hum Reprod 2013;28:2755-64

30 Xu K, Evans DB, Carrin G, et al. Protecting households from catastrophic health spending. Health Aff 2007;26:972-83.

31 Connolly MP, Hoorens S, Chambers GM, et al. The costs and consequences of assisted reproductive technology: an economic perspective. Hum Reprod Update 2010;16:603-13.
32 Chambers GM, Adamson GD, Eijkemans MJC. Acceptable cost for the patient and society. Fertil Steril 2013;100:319-27.

33 Habbema JDF. Is affordable and cost-effective assisted reproductive technology in low-income countries possible? what should we know to answer the question? ESHRE Monographs 2008;2008:21-4.

34 Klitzman R. How much is a child worth? providers' and patients' views and responses concerning ethical and policy challenges in paying for art. PLoS One 2017;12:e0171939.

35 Dyer SJ, Patel M. The economic impact of infertility on women in developing countries - a systematic review. Facts Views Vis Obgyn 2012;4:102-9.

36 Sallam HN. Infertility in developing countries: funding the project. ESHRE Monographs 2008;2008:97-101.

37 Moher D, Shamseer L, Clarke M, et al. Preferred reporting items for systematic review and meta-analysis protocols (PRISMA-P) 2015 statement. Syst Rev 2015;4:1.

38 Moher D, Liberati A, Tetzlaff J, et al. Preferred reporting items for systematic reviews and meta-analyses: the PRISMA statement. PLoS Med 2009;6:e1000097.

39 Lewis M. Informal payments and the financing of health care in developing and transition countries. Health Aff 2007;26:984-97.

40 Mertz M. How to tackle the conundrum of quality appraisal in systematic reviews of normative literature/information? Analysing the problems of three possible strategies (translation of a German paper). BMC Med Ethics 2019;20:81.

41 Zingg W, Castro-Sanchez E, Secci FV, et al. Innovative tools for quality assessment: integrated quality criteria for review of multiple study designs (ICROMS). Public Health 2016;133:19-37.

42 WHO. Guidelines on core components of infection prevention and control programmes at the national and acute health care facility level [Internet], 2016. Available: http://www.ncbi.nlm.nih.gov/books/ NBK401773/ [Accessed 22 Jun 2020]. 